\title{
Refractive Error
}

National Cancer Institute

\section{Source}

National Cancer Institute. Refractive Error. NCI Thesaurus. Code C87145.

A defect in the focusing of light on the retina as in astigmatism, myopia, or hyperopia. 